\title{
Connectivity in Rett
}

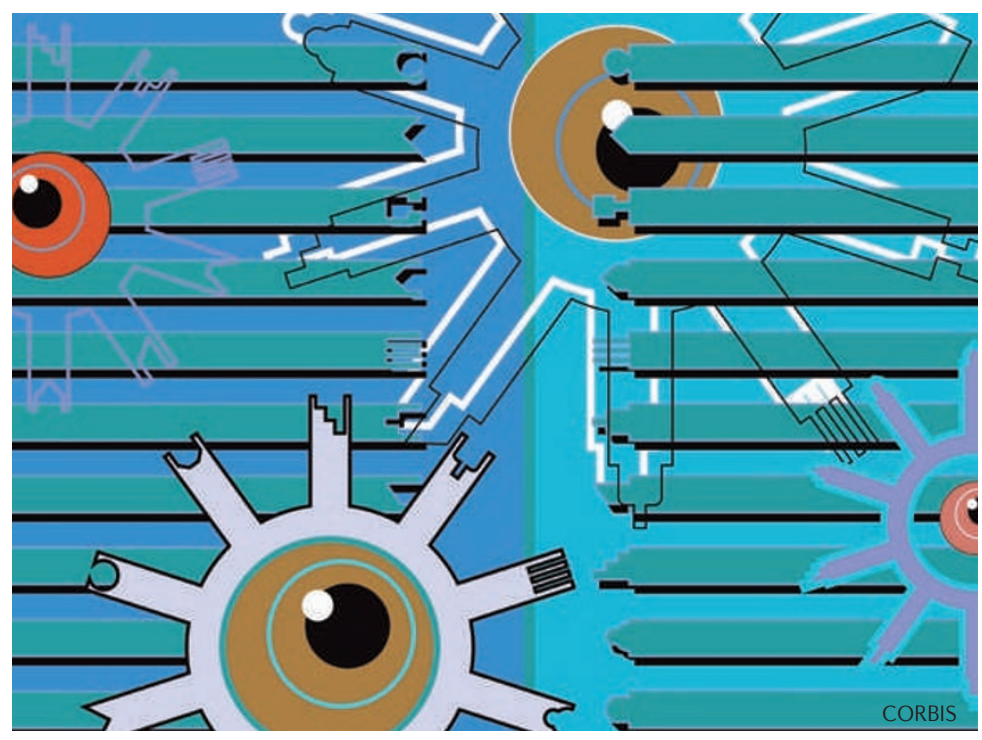

Rett syndrome is a neurodevelopmental disorder that manifests in early childhood and is caused by mutations in the gene encoding methyl-CpG-binding protein 2 (MECP2). Previous studies have shown that synaptic input to layer 5 pyramidal neurons is abnormal in Mecp2-null mice, which have similar symptoms to children with Rett syndrome, namely motor abnormalities and profound impairment of learning and memory. Now, Dani and Nelson show that Mecp2-null mice form only half of the normal number of connections onto layer 5 (L5) pyramidal neurons.

The authors made paired recordings in cortical slices from wild-type and Mecp2-null mice before (2-3-week-old mice) and after (4-5-week-old mice) the onset of symptoms. In contrast to previous studies at hippocampal synapses, the authors did not detect notable differences in the induction characteristics of long-term potentiation (LTP) between L5 pyramid-pyramid synapses of wild-type and Mecp2null mice before and after the onset of symptoms. However, baseline synaptic transmission was altered: the mean amplitude of the excitatory postsynaptic potential (EPSP) at L5 pyramid-pyramid synapses of 4-week-old Mecp2-null mice was only half of that at wild-type synapses. Furthermore, the quantal content of released presynaptic vesicles during a train of EPSPs was lower than normal at wild-type synapses but not at the synapses of Mecp2-null mice. This suggests that reduced quantal content at the synapses of symptomatic Mecp2-null mice might cause reduced EPSPs.

Further electrophysiological investigations revealed that although $10 \%$ of tested L5 neuron pairs had functional synapses in wild-type slices, only $5 \%$ of synapses in slices of 4-week-old Mecp2-null mice were functionally connected, indicating that mutations in Mecp2 reduce the excitatory connectivity in local cortical microcircuits. This observation might explain prior findings of impaired LTP in Mecp2-null mice, as decreased connectivity might make it more difficult to induce LTP using standard extracellular stimulation.

This study indicates that altered connectivity in neocortical circuits rather than impaired synaptic potentiation might underlie the learning and cognitive disabilities associated with Rett syndrome; however, whether decreased connectivity is the primary effect of Mecp 2 mutations remains to be determined.

Claudia Wiedemann

ORIGINAL RESEARCH PAPER Dani, V. S. \& Nelson, S. B. Intact long-term potentiation but reduced connectivity between neocortical layer 5 pyramidal neurons in a mouse model of Rett syndrome. J. Neurosci. 29, 11263-11270 (2009) 